\title{
Facilitating chlamydia testing among young people: a randomised controlled trial in cyberspace
}

\author{
Melissa Kang, ${ }^{1}$ Arlie Rochford, ${ }^{1}$ Rachel Skinner, ${ }^{2}$ Adrian Mindel, ${ }^{3}$ Marianne Webb, ${ }^{4}$ \\ Jenny Peat, ${ }^{5}$ Tim Usherwood ${ }^{1}$
}

- Additional appendices are published online only. To view these files please visit the journal online (http://dx.doi.org/ 10.1136/sextrans-2011050124).

'Discipline of General Practice, Sydney Medical

School-Westmead, University of Sydney, New South Wales,

Australia

${ }^{2}$ Sydney University Discipline of Paediatrics and Child Health, Children's Hospital Westmead, Sydney, Australia

${ }^{3}$ Sexually Transmitted Infections Research Centre, University of Sydney, Sydney, New South Wales, Australia

${ }^{4}$ Inspire Foundation, Sydney, New South Wales, Australia ${ }^{5} \mathrm{New}$ South Wales, Australia

\section{Correspondence to} Dr Melissa Kang, Discipline of General Practice, Sydney Medical School-Westmead, PO Box 154, Westmead, NSW 2145, Australia;

melissa.kang@sydney.edu.au

Accepted 4 June 2012

Published Online First

4 July 2012

\begin{abstract}
Objectives Chlamydia notifications have been rising in Australia for over a decade and are highest in young people. This study aimed to evaluate the impact of an internet-based intervention on chlamydia testing among young people $16-25$ years.
\end{abstract}

Methods In this randomised controlled trial, recruitment, data collection, study interventions and follow-up occurred entirely in cyberspace, facilitated by a website. Eligible participants were aged $16-25$ years and resided in Australia. The intervention group received personalised emails inviting interaction about chlamydia testing, while the control group received regular impersonal emails.

Primary outcome was self-reported chlamydia testing at 6-month follow-up; secondary outcomes were condom use and changes in knowledge and attitudes.

Results 704 young people completed baseline information, 40 were excluded and five withdrew prior to follow-up. The follow-up rate was $47.3 \%$ overall. In the intervention group, $40.6 \%$ (95\% Cl $30.7 \%$ to $51.1 \%$ ) reported having had a chlamydia test at follow-up compared with $31.0 \%$ (95\% Cl $24.8 \%$ to $37.2 \%$ ) in the control group ( $p=0.07)$. A per-protocol analysis found that those who engaged in email interaction were more likely to report chlamydia test uptake compared with those in the control group $(52.5 \%, 95 \% \mathrm{Cl} 39.3$ to $65.4 \%$ cf $31.0 \%, 95 \% \mathrm{Cl} 24.8 \%$ to $37.2 \%, p=0.002$ ). There were no differences in secondary outcomes between groups.

Conclusions This is the first randomised controlled trial undertaken in cyberspace to promote chlamydia testing. E-technology may be useful in promoting chlamydia testing and healthcare seeking behaviour in young people.

\section{INTRODUCTION}

Among the sexually transmissible infections (STIs) other than HIV, chlamydia causes the greatest burden of disease globally. ${ }^{1}$ In Australia, notification rates for genital chlamydia infection have steadily increased over the past decade and are highest in women and men aged $15-24$ years. ${ }^{2}$ In 2011, notification rates were 1443.3 per 100000 among 15-19-year-olds and 1801.4 per 100000 among 20-24-year-olds. ${ }^{2}$ Australia's first National STI strategy 2005-2008 identified young people aged $16-25$ years as a target group for chlamydia control and prevention. ${ }^{3}$ This study was one of several pilot projects to inform a national screening programme ${ }^{4}$ and aimed to evaluate an internetbased intervention to increase chlamydia testing.
Australian young people experience several barriers to care, including lack of knowledge about services available and how to access them, concerns about confidentiality, embarrassment, cost and transport. ${ }^{5}$ However, most young people are familiar with and access general practice. ${ }^{6}$ An analysis of general practice encounters across Australia from 2000 to 2007 found that the rate of chlamydia testing among 15-24-year-old patients was only 13.2 per 1000 encounters. ${ }^{7}$ Chlamydia testing rates among young people are higher in sexual health and family planning clinics, ${ }^{8}$ but unlike general practices, these are not located in many rural and some urban areas. Thus, the majority of the youth population who might be at risk are unlikely to be tested for chlamydia.

Over $85 \%$ of Australian young people access the internet each year ${ }^{9}$ and young people find it useful for seeking help around sensitive issues. Furthermore, men are as likely as women to use the internet to seek help..$^{9-11}$ A study in Switzerland suggested that the internet can facilitate access to healthcare through the use of tailored information provided to individuals in response to questions asked via a website. ${ }^{12}$

This study used the internet to engage sexually active young people aged $16-25$ years residing in Australia, in confidential personalised email interactions with a clinician, with the goal of facilitating their access to primary health services for chlamydia testing. The primary aim of this study was to evaluate the impact of this intervention on chlamydia testing when compared with a control intervention (monthly impersonal emails for 6 months).

\section{METHODS \\ Study location and population}

This was a randomised controlled trial (RCT) with 1:2 randomisation. Eligible participants were aged 16-25 years residing in Australia who had had penetrative sexual intercourse and who provided a valid email address. Eligibility was determined by self-report of these criteria.

The study setting was cyberspace. A website (http://www.getcluedup.com.au) was the vehicle for accessing the intervention and was developed in consultation with 20 youth consultants (16-25 years) who were recruited through professional and collegiate networks. Website content included information about chlamydia and testing but also addressed known gaps in knowledge ${ }^{13}$ and barriers to seeking help ${ }^{5}$ and provided service 
directories and links (see web appendix 1). The website went live in March 2007 with recruitment into the study commencing simultaneously. Recruitment ceased in January 2008, but the website remains live. The website was promoted via paid advertising, existing youth websites, social networking sites and opportunistic media interviews. Google Analytics was used to monitor website traffic.

The website invited eligible visitors to participate in the study via a homepage banner and clickable tiles on the other webpages. These links took visitors to the participant information statement. Potential participants then entered a current email address and ticked a consent box. This third step took them to the baseline questionnaire housed within the website. The baseline questionnaire collected data on demographics, education/occupation, substance use, sexual history, previous STIs, knowledge about chlamydia and attitudes to chlamydia testing. Participants could go into a draw for an iPod $\AA$ or a music store voucher if they completed all elements of the study.

\section{Interventions}

The intervention group received personalised emails from a clinician (sexual health nurse or doctor). A 'personalised email' was sent from the clinician's mailbox, included the clinician's name and position, and contained a link to their staff profile on the University of Sydney's website. The email thanked the young person for their participation and said that the clinician would like to 'chat about chlamydia and getting tested'. The participant was invited to ask questions and prompted with questions about testing knowledge. Young people who responded were then engaged appropriately: advice depended on the questions asked. Non-responders were sent weekly emails for 3 months and then monthly emails for another 3 months. All email communication to non-responders in this group remained personalised, as described above.

Participants assigned to the control group received an email sent from the project mailbox ('Clued Up'), was signed 'The Clued Up Research Team' and did not mention a clinician by name. These emails thanked the young person for participation and stated that they would be sent a reminder email about their participation in the study every month for 5 months and a final questionnaire in 6 months. These emails were intended to enhance retention and completion of the final questionnaire but were not personalised. There was no interaction and no clinical advice provided.

Web appendix 2 gives examples of email interactions.

\section{Outcomes}

Follow-up took place 6 months after enrolment. Data were collected via an online questionnaire using similar questions to baseline on knowledge, attitudes, sexual history and chlamydia testing in the past 6 months. This questionnaire was accessed via a link sent by email from the clinician. Weekly email reminders were sent for 3 months to non-responders.

The primary outcome was self-report of having had a chlamydia test within the past 6 months. Secondary outcomes were changes in knowledge about chlamydia, attitudes towards chlamydia testing and frequency of condom use.

\section{Sample size}

A minimum of 320 participants in each group was required to detect at least an $8 \%$ positive difference in testing rates in the intervention versus control group with a power of $90 \%$ and significance taken at 0.05 . A loss to follow-up of $10 \%$ in the intervention group and $50 \%$ in the control group was antici- pated, giving a target sample size of 1000 (360 intervention and 640 control).

\section{Randomisation}

Allocation to intervention or control groups was done on a 1:2 ratio based on the anticipated loss to follow-up described above. For allocation, a computer-generated random number (1, 2 or 3 ) was obtained by the same clinicians who sent emails to study participants. The number ' 1 ' allocated the young person to the intervention group and ' 2 ' or ' 3 ' to the control group. Allocation took place each time a young person was deemed eligible (downloaded data showed email address, eligible age, an Australian postcode and ticked 'Yes' to having had intercourse) without any other information about the participant being known.

\section{Analysis and statistical methods}

Both intention-to-treat (ITT) and per-protocol (PP) analyses were performed. Only a proportion of young people in the intervention group interacted with the clinician as per the study protocol, that is, engaged. Engagement was defined as having had a minimum of one response of any type from the young person within 3 months from enrolment. The intervention was only deliverable when engagement occurred, where it did not occur, there was a 'failure to start the intervention'. ${ }^{14}$ Thus, comparisons between the engaged group and the control group constituted the PP analysis. We propose that the PP analysis provides an 'explanatory investigation of efficacy ${ }^{\text {'14 }}$ and provides useful information.

Statistical analysis was performed with SPSS V.16 with the individual as the unit of analysis. Proportions are presented with 95\% CIs. To assess differences at baseline between the intervention (all), engaged and control groups, independent samples' $t$ tests for the continuous variables and $\chi^{2}$ tests for the categorical variables were performed. $\chi^{2}$ Analysis was used to assess the statistical significance of differences in the primary outcome and in condom use between groups at follow-up. To adjust follow-up values for baseline values, analysis of covariance was used for knowledge questions and logistic regression for binary outcome measures.

The study was approved by the University of Sydney Human Research Ethics Committee and enrolled in the Australian New Zealand Clinical Trials Registry (ACTRN12607000582459).

\section{RESULTS}

\section{Website traffic}

Reliable data on website traffic for the first 3 months (March to May 2007) were unavailable due to technical problems. Traffic between June 2007 and January 2008 ranged from 2030 to 3584 unique visitors per month (see web appendix 3).

\section{Sample}

Seven hundred and four young people completed baseline information between March 2007 and January 2008 and were randomised. Recruitment was then stopped because of project timelines and budget constraints. Forty young people were subsequently excluded because their email addresses were invalid (all sent emails bounced). Five withdrew prior to follow-up. Three hundred and forty-seven participants did not respond to the 6-month follow-up request. Thus, 312 were included in the ITT analysis (see figure 1).

We compared baseline information between intervention (all), engaged and control groups. Of the baseline sample of 664 
Figure 1 Participant flowchart.

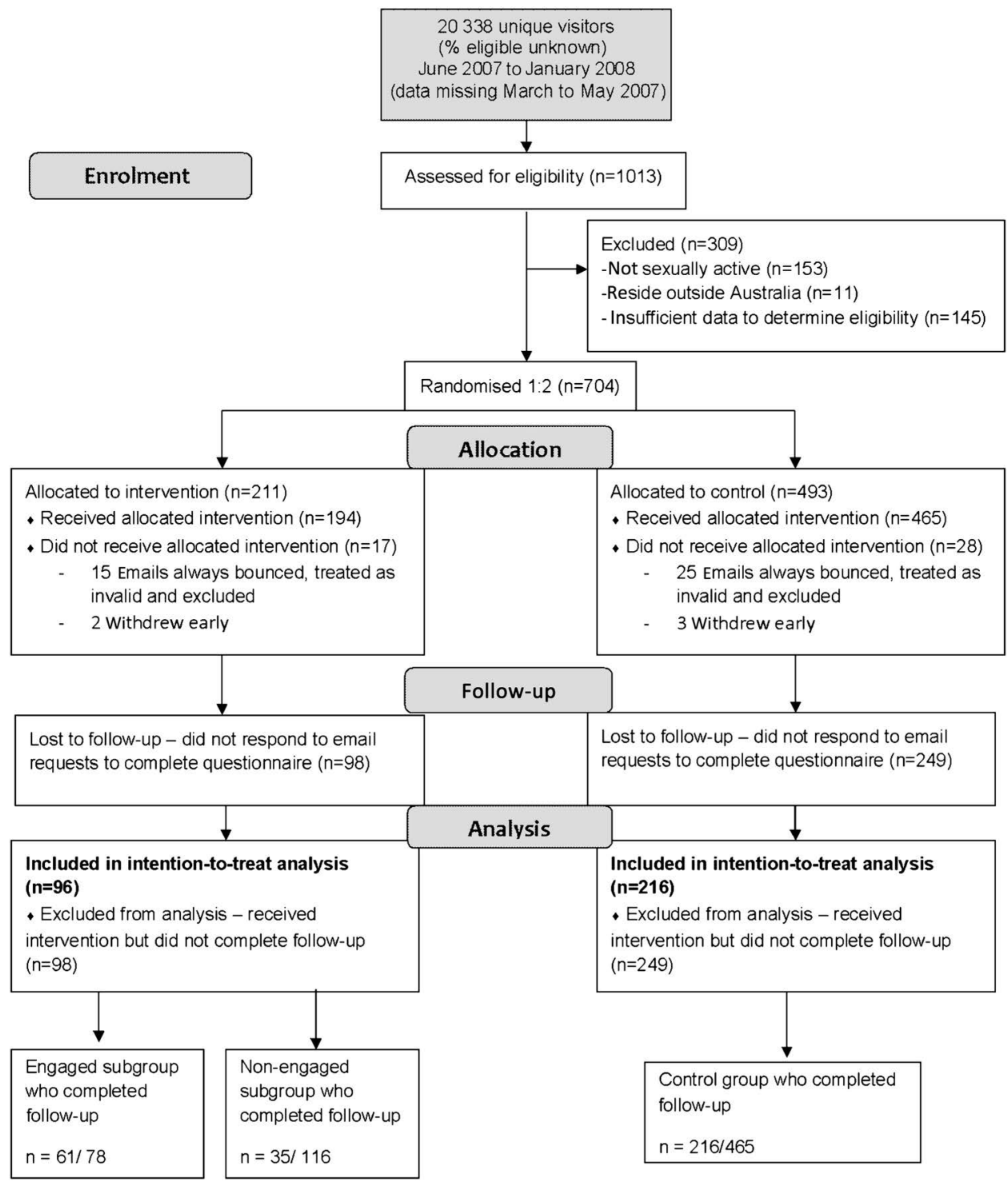

young people, $78.2 \%$ were were. The mean age of female participants (20.0 years) was significantly lower than the mean age of male participants (21.5 years), $p<0.0001$. There was a small significant difference between the engaged and whole intervention groups with respect to being born overseas. Those who engaged were more likely to have been born in Australia. However, of those born overseas in both groups, most were born in English-speaking countries. Table 1 describes their demographic and sexual history characteristics. At baseline, 111/664 $(16.7 \%$, $95 \%$ CI $13.9 \%$ to $19.6 \%)$ reported having ever had chlamydia.

The follow-up rate was $47.3 \%(312 / 659)$ overall. There were no differences in demographic (mean age, sex) or baseline sexual history characteristics (number of sexual partners ever, condom use) between those who completed follow-up questionnaires and those who did not (data not shown). There was no difference in follow-up rate between the intervention $(49.5 \%$; 96/194) and control $(46.5 \% ; 216 / 465)$ groups; however, follow-up rate for the 'engaged' group (78.2\%; 61/78) was significantly higher than for the 'non-engaged' group (30.7\%; 35/114), $\mathrm{p}<0.0001$, and for the control group (46.6\%, 216/464), $\mathrm{p}<0.0001$.

Table 2 presents the primary outcome measurement in the intervention and control groups (ITT) and in the engaged and control groups (PP analysis). Given the loss to follow-up at 6 months is a problem for ITT analysis, we also conducted a sensitivity analysis to examine the possible effects. A higher proportion of young people in the intervention group reported a chlamydia test compared with the control group ( $40.6 \%$ vs $31.0 \%)$ but this was not significant. However, the difference between the engaged intervention and control groups was significant $(52.5 \%$ and $31.0 \%$, respectively, $\mathrm{p}=0.002)$.

Of those who reported having had a chlamydia test at followup, a total of $14 / 99$ ( $14.1 \%$, 95\% CI $7.95 \%$ to $22.6 \%$ ) young people reported their tests were positive for chlamydia. Three were from the engaged group and 11 from the control group.

The proportion of young people who reported using condoms every time they had sex increased in the engaged and the control groups; however, this increase was not significant. At baseline, $12 / 61(19.7 \%)$ in the engaged group and $27 / 216(12.5 \%)$ in the control group reported using condoms always, at follow-up these proportions were 20/61 (32.8\%) and 59/216 (27.3\%), respectively $(p=0.30)$.

There was no change in knowledge between baseline and follow-up; however, baseline knowledge was high. The proportion of young people in total $(n=312)$ who answered each of the seven questions correctly ranged from $77.6 \%$ to $95.8 \%$ at 
Table 1 Baseline demographic information, sexual history and substance use

\begin{tabular}{|c|c|c|c|c|c|}
\hline & $\begin{array}{l}\text { Intervention } \\
\text { (all), } n=196\end{array}$ & $\begin{array}{l}\text { Intervention } \\
\text { (engaged), } \mathrm{n}=79\end{array}$ & $\begin{array}{l}\text { Control, } \\
n=468\end{array}$ & $\begin{array}{l}\text { Intervention (all) versus } \\
\text { engaged, } p \text { value }\end{array}$ & $\begin{array}{l}\text { Intervention all versus } \\
\text { control, p Value, }\end{array}$ \\
\hline Mean age (years) & 20.5 & 20.7 & 20.3 & 0.50 & 0.42 \\
\hline Female (\%) & $154(78.6)$ & $65(82.3)$ & $365(78.0)$ & 0.46 & 0.97 \\
\hline Aboriginal/Torres Strait Islander (\%) & $4(2.0)$ & $2(2.5)$ & $14(3.0)$ & 1.00 & 0.70 \\
\hline Region of residence & & & & 0.29 & 0.21 \\
\hline Major city (\%) & $147(75.0)$ & $54(68.4)$ & $317(67.7)$ & & \\
\hline Remote (\%) & $0(0.0)$ & $0(0.0)$ & $4(0.9)$ & & \\
\hline Very remote (\%) & $0(0.0)$ & $0(0.0)$ & $0(0.0)$ & & \\
\hline Occupation & & & & 0.70 & 0.42 \\
\hline School (\%) & $35(17.9)$ & $11(13.9)$ & $98(20.9)$ & & \\
\hline University/tertiary (\%) & $60(30.6)$ & $26(32.9)$ & $128(27.4)$ & & \\
\hline Full time work (\%) & $62(31.6)$ & $27(34.2)$ & $149(31.8)$ & & \\
\hline Other $(\%)$ & $2(1.0)$ & $1(1.3)$ & $7(1.5)$ & & \\
\hline \multicolumn{6}{|l|}{ Sexual history } \\
\hline Mean age of first intercourse (years) & 16.2 & 16.5 & 16.3 & 0.15 & 0.42 \\
\hline Mean no. of sexual partners ever & 3.1 & 3.2 & 3.1 & 0.83 & 0.57 \\
\hline Mean no. of sexual partners last 12 months & 2.0 & 1.9 & 2.0 & 0.31 & 0.88 \\
\hline Use condoms always (\%) & $41(20.9)$ & $20(25.3)$ & $100(21.4)$ & 0.62 & 0.95 \\
\hline Had chlamydia test in past 6 months $(\%)$ & $60(30.6)$ & $26(32.9)$ & $127(27.1)$ & 0.49 & 0.57 \\
\hline Had a previous diagnosis of chlamydia (\%) & $26(13.3)$ & $13(16.5)$ & $85(18.2)$ & 0.56 & 0.21 \\
\hline \multicolumn{6}{|l|}{ Substance use history } \\
\hline Daily smoker past 12 months (\%) & $37(18.9)$ & $17(21.5)$ & $102(21.8)$ & 0.80 & 0.44 \\
\hline Drink 5 or more standard drinks at a time (\%) past 2 weeks & $93(47.4)$ & $47(59.5)$ & $201(42.9)$ & 0.67 & 0.95 \\
\hline
\end{tabular}

baseline and 80.8 to $97.4 \%$ at follow-up. There was no difference between change in knowledge in the intervention and control groups or in the engaged and control groups. There were no attitude differences between the intervention and control or engaged and control groups at follow-up. The list of knowledge questions and attitude statements are shown in web appendix 4.

Table 2 Self-reported chlamydia test in the past 6 months by group allocation (including sensitivity analysis)

\begin{tabular}{lrc}
\hline & $\% ; 95 \% \mathbf{C l}$ & p Value \\
\hline $\begin{array}{l}\text { Test uptake in those randomised and } \\
\text { followed up (intention-to-treat population) }\end{array}$ & 0.07 \\
$\quad$ Intervention & $39 / 96(40.6 ; 30.7-51.1)$ & \\
$\quad$ Control & $67 / 216(31.0 ; 24.8-37.2)$ & \\
$\begin{array}{l}\text { Test uptake in those randomised } \\
\text { who 'engaged' per-protocol }\end{array}$ & 0.002 \\
$\quad$ Engaged & $32 / 61(52.5 ; 39.3-65.4)$ & \\
$\quad$ Control & $67 / 216(31.0 ; 24.8-37.2)$ & \\
Test uptake in all randomised, & & 0.09 \\
assuming all those lost to follow-up & & \\
did not have a test & & \\
$\quad$ Intervention & $39 / 194(20.1 ; 14.7-26.4)$ & \\
$\quad$ Control & $67 / 465(14.4 ; 11.3-17.9)$ & \\
Test uptake in all randomised, & & \\
assuming those lost to follow-up & & \\
all had a test & & \\
$\quad$ Intervention & & \\
$\quad$ Control & $137 / 194(70.6 ; 63.7-76.9)$ & \\
\hline
\end{tabular}

The amount of clinician time required to respond to emails for participants who engaged in interaction was low (a few minutes per email). Responses usually provided information about what testing involved, confidentiality and services available. Some young people asked about other screening tests such as Pap smears, and some were interested in other STIs.

\section{DISCUSSION}

To our knowledge, this is the first RCT of an intervention to increase chlamydia testing implemented entirely in cyberspace. Young people who engaged in email interaction with a clinician were more likely to report having a chlamydia test after 6 months than young people who were not offered such contact. Knowledge, attitudes and condom use did not change.

Strengths of this study include originality, youth consultant advice and experimental rigour despite the uncontrollable study environment. Clinicians provided clear information about testing, treatment and relevant services rather than messages about 'risk' that could provoke fear. They answered young people's questions regardless of topic, thus feeling listened-to might have generated confidence in seeking healthcare.

There were several limitations. Website reach was modest and target sample size was not achieved due to project constraints. This combined with loss to follow-up reduced the power of the study. The main outcome measure was based on self-report of testing that could not be verified. Overall followup rate was just under $50 \%$; however, of those who engaged, $61 / 78(78.2 \%)$ completed follow-up. Of the intervention group, 
$59.4 \%$ did not engage. Although there were no measurable differences in attitudes between these subgroups, it is possible that those who engaged were more concerned about chlamydia, more engaged with the health system or wanted to please the clinician or avoid embarrassment. Internet-based trials have high attrition rates, especially when there is no clinician contact at the outset. ${ }^{15}$ Attrition can occur either at uptake of an intervention or with failure to complete followup. This introduces dilemmas with analysing ITT populations in exactly the way we found. To improve validity, it has been suggested that individuals who do not take up an intervention be removed and that the remaining participants in the intervention group undergo a second randomisation process. ${ }^{16}$ Given our time frame, this was not feasible. Most internetbased RCTs have evaluated therapeutic interventions, such as pain management, chronic illness or mental health treatments. ${ }^{15-18}$ It is possible that participants who are symptomatic are more likely to take up an intervention but also to dropout if no benefit is perceived. Our trial involved an intervention that was not therapeutic and targeted a behaviour change for an asymptomatic condition. We might therefore expect a higher dropout rate compared with therapeutic interventions but this was not the case. Nevertheless, we must be cautious about how to interpret our findings.

Other studies have used e-technology to increase chlamydia testing among young people. A RCT using SMS and email delivered sexual health messages to 994 young people who were recruited from a music festival. The study found that at 12 month follow-up, young women in the intervention group were significantly more likely to report Chlamydia testing than those in the control group. Follow-up rates were substantially lower than ours (34\%). ${ }^{19}$

Strategies combining the internet with home-based chlamydia testing have been evaluated. In Sweden, $62.5 \%$ of kits requested over the internet were returned for testing, ${ }^{20}$ in the USA $32.4 \%$ of women ${ }^{21}$ and $31 \%$ of men ${ }^{22}$ returned samples and in the Netherlands, $20 \%$ of the invitees requested testing kits and $16 \%$ returned samples. ${ }^{23}$ Although our sample size was small, the fact that over $50 \%$ of young people who engaged in email interaction reported chlamydia testing at follow-up without the convenience of a home testing kit suggests that email interaction could help facilitate access to health services more broadly.

We found no significant changes in knowledge, attitudes or condom use. However, baseline knowledge was high and might have been positively impacted simply from navigating the website. That reported condom use was low and did not increase significantly highlights the complexities of this behaviour and relationship dynamics at the point of sexual encounter. ${ }^{24}$ Furthermore, heterosexual couples using other contraceptive methods are less likely to use condoms ${ }^{25}$ or pregnancy might be desired.

Cyberspace research presents methodological challenges. Participation rates are not measurable with unknown denominators, and external validity is difficult to ascertain. ${ }^{26}$ Website traffic data do not provide demographic information that would help determine eligibility or generalisability. Technology also changes rapidly. Members of our Youth Advisory Committee developed a MySpace page to promote our website and Facebook had not reached Australia. Between 2009 and 2011, Facebook overtook MySpace in number of unique visitors, and email has been overtaken or replaced by Facebook chatting, immediate messaging and texting. ${ }^{27}$

Advantages include the potential to 'scale up' to achieve greater reach for minimal additional cost: our greatest cost was

\section{Key messages}

Young people who engaged with clinicians online were more likely to access chlamydia testing than those who did not engage.

- Conducting studies in cyberspace is novel and presents challenges to the conventional frameworks for evaluating and interpreting scientific data.

- The internet can be a useful vehicle for sexual health promotion and an adjunct to screening strategies.

website development. Social networking may provide cheaper alternatives to paid advertising. The study clinicians could easily have managed a greater volume of emails at no additional cost. The internet has the potential to reach hard-to-reach populations, and the intervention itself could be transferable to other health issues.

Potential ethical issues include email security, the misuse of online communication and ownership of personal information posted on the world wide web. ${ }^{28}$ While all data entered by participants in our study were secure and we were not aware of any ethical concerns, it is possible that concerns about privacy and security influenced young people's decisions to participate in the study or to disclose personal information.

Our study has implications for further research. Cost-benefit analyses of a range of screening strategies would be useful for informing a broader screening programme. Qualitative research would help us understand more about why some young people engaged and others did not and their experiences of internetbased clinical interaction. A qualitative study with volunteers from our study has been undertaken.

Chlamydia is a common curable sexually transmitted infection that can have potentially serious consequences if undetected and untreated. E-technology has the potential to be a useful adjunct to a population-based screening programme.

Acknowledgements We would like to thank our Youth Advisors and all the young people who participated in the study.

Contributors MK prepared the first draft of the manuscript and completed revisions after discussion with the entire research team. MK, RS, AM, MW and TU participated in study design. MK wrote the content of the website. AR, RS, AM and MW reviewed website content. MK and MW recruited and facilitated the Youth Advisory Committee. AR and MK recruited study participants, delivered the intervention and collected all data. MK, AR, JP and TU completed data analysis. All authors assisted with interpretation of findings.

Funding This study was funded by the Australian Department of Health and Ageing as part of the Chlamydia Targeted Grants Programme.

\section{Competing interests None.}

Ethics approval Ethics approval was provided by the University of Sydney Human Research Ethics Committee.

Provenance and peer review Not commissioned; externally peer reviewed.

\section{REFERENCES}

1. World Health Organisation 2008. The global burden of disease: 2004 update. Switzerland: WHO, 2008.

2. Australian Government Department of Health and Ageing. National Notifiable Diseases Surveillance System. Commonwealth of Australia. http://www9.health.gov. $\mathrm{au} / \mathrm{cda} /$ Source/Rpt 2 sel.cfm (accessed Nov 2011).

3. Australian Government Department of Health and Ageing. National Sexually Transmissible Infections Strategy 2005-2008. Canberra: Commonwealth of Australia, 2005. http://www.health.gov.au/internet/main/publishing.nst/Content/ 0333DF52D0E2F3EDCA25702A0025132F/\$File/sti_strategy.pdf (accessed Nov 2011) 
4. Minister for Health and Ageing. Chlamydia Projects to Target High Risk Groups [Media Release]. Commonwealth Department of Health and Ageing, AB115/06. Canberra: ACT, 2006. http://www.health.gov.au/internet/ministers/publishing.nsf/ Content/9FCD4CEED36DEBODCA2571C4000F9DA8/\$File/abb115.pdf laccessed Nov 2011)

5. Booth ML, Bernard D, Quine S, et al. Access to health care among Australian adolescents: young people's perspectives and their socio-demographic distribution $J$ Adolesc Health 2004;34:97-103.

6. Kang M, Sanci LA. Primary health care for young people in Australia Int J Adolesc Med Health 2007;19:229-34.

7. Sawleshwarkar $\mathbf{S}$, Britt $\mathrm{H}$, Harrison C, et al. Chlamydia testing in general practice in Australia. Sex Health 2010;7:484-90.

8. National Centre in HIV Epidemiology and Clinical Research. HIV/AIDs, Viral Hepatitis and Sexually Transmissible Infections in Australia Annual Surveillance Report 2009. Sydney, NSW: National Centre in HIV Epidemiology and Clinical Research, The University of New South Wales. http://www.access-study.org/images/documents/ asr2009 updated 2 seepage109.pdf (accessed Nov 2011).

9. Nicholas J, Oliver $\overline{\mathrm{K}}$, Lee $\mathrm{K}$, et al. Help-seeking behaviour and the internet: an investigation among Australian adolescents. A eJAMH 2004;3:16-23.

10. Gould MS, Munfakh JLH, Lubell K, et al. Seeking help from the internet during adolescence. J Am Acad Child Adolesc Psychiatry 2002;41:1182-9.

11. Hillier L, Turner A, Mitchell A. Writing Themselves In Again: 6 years on. The second National Report on the Sexual Health and Well-being of Same-Sex Attracted Young People in Australia. Monograph Series No. 50. Melbourne: Australian Research Centre in Sex, Health and Society, La Trobe University, 2004.

12. Michaud PA, Colom P. Implementation and evaluation of an Internet health site for adolescents in Switzerland. J Adolesc Health 2003;33:287-90.

13. Smith A, Agius P, Mitchell A, et al. Secondary Students and sexual health 2008. Monograph Series No. 70. Melbourne: Australian Research Centre in Sex, Health and Society, La Trobe University, 2009.

14. Hollis $\mathbf{S}$, Campbell F. What is meant by intention to treat analysis? Survey of published randomised controlled trials. BMJ 1999;319:670-4.

15. Bender JL, Radhakrishnan A, Diorio C, et al. Can pain be managed through the Internet? A systematic review of randomized controlled trials. Pain 2011:152:1740-50.

16. Eysenbach G. The law of attrition. J Med Internet Res 2005;7:e11.
17. Andersson G, Cuijpers P. Internet-based and other computerized psychological treatments for adult depression: a meta-analysis. Cogn Behav Ther 2009;38(Suppl 4):196-205.

18. Rosser BA, Vowles KE, Keogh E, et al. Technologically-assisted behaviour change: a systematic review of studies of novel technologies for the management of chronic illness. J Telemed Telecare 2009:15:327-38.

19. Lim MSC, Hocking JS, Aitken CK, et al. A randomized controlled trial of text and email messaging for sexual health promotion to young people. J Epidemiol Community Health 2012:66:69-74.

20. Novak DP, Karlsson RB. Simplifying chlamydia testing: an innovative Chlamydia trachomatis testing approach using the internet and a home sampling strategy: population based study. Sex Transm Infect 2006;82:142-7.

21. Gaydos CA, Barnes M, Aumakhan B, et al. Can e-technology through the internet be used as a new tool to address the Chlamydia trachomatis epidemic through home sampling and vaginal swabs? Sex Transm Dis 2009:36:577-80.

22. Chai SJ, Aumakhan B, Barnes M, et al. Internet-based screening for sexually transmitted infections to reach nonclinic populations in the community: risk factors for infection in men. Sex Transm Dis 2010;37:756-63.

23. Greenland KE, Op de Coul ELM, van Bergen JE, et al. Acceptability of the internetbased Chlamydia screening implementation in the Netherlands and insights into nonresponse. Sex Transm Dis 2011;38:467-74.

24. Smith JL, Fenwick J, Skinner R, et al. Young males' perspectives on pregnancy, fatherhood and condom use: where does responsibility for birth control lie? Sex Reprod Healthc 2011;2:37-42.

25. Smith J, Skinner SR, Fenwick J. How Australian female adolescents prioritize pregnancy protection: a grounded theory study of contraceptive Histories. J Adolesc Res 2011;5:617-44.

26. Mustanski BM. Methodological issues in online sexual health research. In: Liamputtong P, ed. Health Research In Cyberspace: Methodological, Personal and Practical Issues. New York: Nova Science Publishers, 2006:121-32.

27. Boyar R, Levine D, Zensius N. TECHsex USA: Youth Sexuality and Reproductive Health in the Digital Age. Oakland, CA: ISIS, Inc, 2011.

28. Liamputtong P. Qualitative cyber research: an introduction. In: Liamputtong P, ed Health Research In Cyberspace: Methodological, Personal and Practical Issues. New York: Nova Science Publishers, 2006:1-24 